\title{
DOES THE SENSE OF RELATIONAL ENTITLEMENT MEDIATE THE ASSOCIATION BETWEEN NARCISSISM AND COUPLE CONFLICT?
}

\author{
Octav Sorin Candel \\ Department of Psychology, Faculty of Psychology and Educational Sciences, Alexandru Ioan Cuza \\ University of Iasi (Romania)
}

\begin{abstract}
Previous literature pointed out that narcissism affects the quality of interpersonal relationships. It has a negative impact on the functioning of the romantic dyads, determining higher levels of conflict and the use of maladaptive conflict resolution styles. However, the mechanisms linking narcissism and couple conflict are not sufficiently explored. This study expands the literature by examining the mediating role of the sense of relational entitlement. Participants in this study were 493 individuals $(52.1 \%$ women, M age $=22.39$ years old) who were in a romantic relationship during the study ( $\mathrm{M}$ relationship length $=26$ months). The results indicated that some forms of relational entitlement mediated the link between narcissism and couple conflict. A person's narcissism was related to their level of couple conflict, excessive and assertive entitlement. Both types of entitlement were related to conflict but in opposite directions. Higher excessive entitlement was associated with higher conflict, while higher assertive entitlement was associated with lower conflict. The indirect effects through both types of entitlement were significant. Restricted entitlement was not associated with narcissism or couple conflict. This study showed that although narcissism can lead to higher levels of relational entitlement and conflict, not all forms of entitlement negatively contributed to couple conflict.
\end{abstract}

Keywords: Romantic relationships, relational entitlement, narcissism, conflict.

\section{Introduction}

Previous literature linked narcissism with a slew of interpersonal difficulties. Among these, one crucial negative outcome is couple conflict (Keller et al., 2014). Narcissists feel superior, are more aggressive, and have more chances to get involved in hostile behaviors toward their partners (Keller et al., 2014). Although the level of aggression can be an important mechanism that explains this relationship, other pathways were less explored. With this study, we propose a novel mediator of the link between narcissism and couple conflict. One's sense of entitlement can be seen as a facet of narcissism but the two do no completely overlap (Campbell et al., 2004). Thus, entitlement was used as a standalone concept and in recent years, it found its application in various life domains, including the functioning of romantic relationships (Tolmacz \& Mikulincer, 2011). A model by Tolmacz and Mikulincer (2011) proposes that people can show three types of a sense of relational entitlement (SRE). People with excessive SRE consider that their needs deserve to be satisfied regardless of the other's feelings. People with restricted SRE have a low sense of autonomy and prefer not to express their needs. Finally, the individuals with an assertive SRE make more realistic evaluations of what they are entitled to. Previous studies linked narcissism and SRE, and general entitlement and conflict (Harvey \& Martinko, 2009; Tolmacz \& Mikulincer, 2011). However, none tested these associations in a romantic domain and none used the sense of relational entitlement. In this study, we hypothesized that: (1) higher narcissism is related to higher SRE and to higher conflict; (2) higher SRE is related to higher conflict, and (3) SRE mediates the association between narcissism and conflict.

\section{Method}

\subsection{Participants}

The sample for this study consisted of 493 individuals $\left(52.1 \%\right.$ women, $M_{\text {age }}=22.39$ years old $)$ who were in a romantic relationship during the study ( $M_{\text {relationship length }}=26$ months). All of them were students and were recruited by the researcher from a Romanian University. Their participation was voluntary. They completed the scales using an online platform and received course credit for their participation. 


\subsection{Instruments}

Single-Item Narcissism Scale (SINS; Konrath, Meier, \& Bushman, 2014). The scale was used to globally self-evaluate the level of narcissism. The single item scale ("To what extent do you agree with this statement; "I am a narcissist [Note: The word 'narcissist' means egotistical, self-focused, and vain]"), measured on a scale from 1 (not very true of me) to 7 (very true of me), has the advantage of being more time effective and less resource consuming for participants in large studies. Moreover, the authors showed that the scale has good convergent, discriminant and construct validity.

Sense of Relational Entitlement Scale (SRE; Tolmacz \& Mikulincer, 2011). This scale was used to measure the level of relational entitlement. The Romanian version of the scale (Candel, 2018) contains 18 items scored from 1 (not at all) to 7 (very much). Eight items measure excessive entitlement (for the present study, $\alpha=.84)$, seven items measure assertive entitlement $(\alpha=.73)$ and 3 items measure restricted entitlement $(\alpha=.76)$.

Conflict scale (Gordon \& Chan, 2016). To assess couple conflict, we used an instrument containing six items, rated on a 7-point scale (1 - strongly disagree, 7 - strongly agree). For this study, $\alpha=.85$.

\subsection{Statistical analyses}

The preliminary and correlational analyses were computed using the IBM SPSS 21 software. The hypotheses were verified using the Process macro. Narcissism was introduced as the predictor, couple conflict as the outcome and the three dimensions of relational entitlement were used as parallel mediators. The software allows calculating both direct and indirect effects. Bootstrapped confidence intervals were used to empirically validate the indirect effects. In order to be significant at a $p<.005$, the CI must not include 0 .

\section{Results}

A series of Independent-sample $\mathrm{T}$ tests showed that women, compared to men, have higher scores at excessive $(t=-4.74, p<.001)$ and assertive SRE $(t=-4.87, p<.001)$. The age of the participants significantly and negatively correlated with their level of restricted entitlement $(r=-.10$, $p=.028)$. The length of the relationships did not correlate significantly with the variables of interest. Narcissism correlated significantly and positively with excessive SRE $(r=.14, \mathrm{p}=.002)$, assertive SRE $(r=.15, p=.001)$ and conflict $(r=.15, p=.001)$. Also, conflict correlated significantly and positively with excessive $(r=.62, p<.001)$ and assertive SRE $(r=.14, p=.001)$. We found significant correlations between excessive SRE $(r=.18, p<.001)$ and the other forms of SRE, restricted and assertive $(r=.35$, $p<.001)$.

Figure 1. Mediation model. On the path between Narcissism and Couple Conflict, the first value represents the total effect and the second value represents the direct effect. Note. ${ }^{* * *} p<.001 ;{ }^{* *} p<.01 ;{ }^{*} p<.05$.

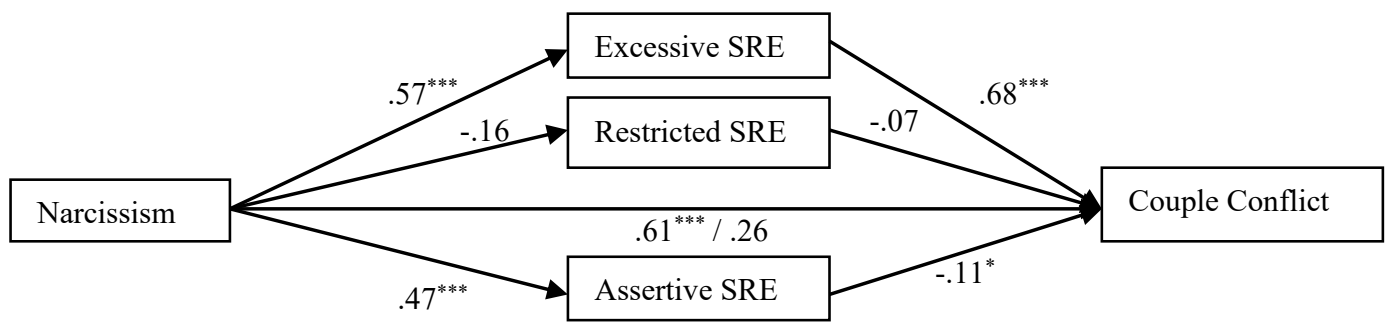

Although restricted entitlement did not correlate significantly with the predictor or the outcome, we decided to keep it in the model in order to test the hypothesized model as we predicted it. We also included the participants' sex and the length of the relationship as control variables. The model explained $40 \%$ of the variation in couple conflict $\left(R_{a d j}^{2}=.40\right)$. We found that both excessive and assertive SRE mediated the relationship between narcissism and couple conflict. More information about the total and direct effects can be found in Figure 1. The indirect effects from narcissism on couple conflict, through excessive SRE $(b=.39,95 \%$ CI $[.19 ; .63])$ and assertive SRE $(b=-.05,95 \%$ CI $[-.11 ;-.01])$ were significant, but in opposite directions.

\section{Discussion}

Firstly, the role of narcissism in interpersonal conflict is well established in the literature and this study only confirms previous findings showing the maladaptive contribution of narcissism (Keller et al., 
2014). Also, the link between narcissism and entitlement was previously explored (Campbell et al., 2004; Tolmacz \& Mikulincer, 2011). This study adds to the existing literature by confirming that narcissism predicts higher levels of excessive and assertive SRE, but is unrelated to restricted SRE. Individuals with high levels of restricted SRE are shy, cautious, and introverted. However, their limited entitlement does not necessarily come from a lack of narcissism. On the contrary, narcissistic individuals seem to show higher levels of excessive and assertive SRE. Narcissists usually put themselves first and consider they are more important than others, and this attitude might also translate into the romantic domain by determining higher levels of SRE. However, it is important how people report to and transmit their expectations to their partners. While individuals with excessive SRE might be unapologetically self-centered and extremely sensible to every transgression, a more assertive type of SRE might determine individuals to be more attentive when expressing their needs. Assertive SRE was related to higher consciousness and self-esteem and not related to attachment issues (Tolmacz \& Mikulincer, 2011). The differences in how these categories of entitled individuals treat their partners might also explain the different ways in which they link narcissism and couple conflict. On the one hand, the narcissists with high levels of excessive SRE demand everything from their partners and respond very negatively when their partners do not respond as they wish. Thus, their excessive needs and expectations can lead to conflicts. On the other hand, the narcissists that are more assertively entitled might express their needs in an adaptive manner, without hurting their partner. Thus, the way they communicate their needs can determine more caring responses from their partners, which might reduce the conflict when it appears.

This study is important in showing the pathways that link narcissism and conflict in romantic couples. However, it is not without its limitations. Firstly, we used a single item to assess narcissism, and although the scale is psychometrically sound, a more complex measure might have provided a more complex view on narcissism. Secondly, the participants were relatively young and their relationships were relatively new. Thus, some of them might still be in the "honeymoon" period, when their satisfaction is high and their conflict is low. A more experienced sample would probably report higher levels of conflict and other relationships might also be different.

With this research, we should that narcissists are generally more entitled to good results in their relationship. Their personality might also lead to more frequent and intense conflicts. However, expressing their needs and expectation from the partners is not inherently bad. When they communicate their needs in a more assertive and adequate way, they might even reduce their level of conflict.

\section{Acknowledgements}

This work was supported by a grant of the "Alexandru Ioan Cuza" University of Iasi, within the Research Grants program, Grant UAIC, code GI-UAIC-2020-01.

\section{References}

Campbell, W. K., Bonacci, A. M., Shelton, J., Exline, J. J., \& Bushman, B. J. (2004). Psychological entitlement: Interpersonal consequences and validation of a self-report measure. Journal of personality assessment, 83(1), 29-45.

Candel, O.S. (2018). Sense of Relational Entitlement - Romanian version. Factor structure and associations with romantic attachment and dyadic satisfaction. In R. Ungureanu \& M. Mocanu (Eds.), Proceedings of CIEA 2018. The Fifth International Conference on Adult Education. Education for values - continuity and context (Iasi, 2018) (79-86). Bologna, Italy: EDlearning.

Gordon, A. M., \& Chen, S. (2016). Do you get where I'm coming from?: Perceived understanding buffers against the negative impact of conflict on relationship satisfaction. Journal of personality and social psychology, 110(2), 239-260.

Harvey, P., \& Martinko, M. J. (2009). An empirical examination of the role of attributions in psychological entitlement and its outcomes. Journal of organizational behavior, 30(4), 459-476.

Keller, P. S., Blincoe, S., Gilbert, L. R., Dewall, C. N., Haak, E. A., \& Widiger, T. (2014). Narcissism in romantic relationships: A dyadic perspective. Journal of social and clinical psychology, 33(1), 25-50.

Konrath, S., Meier, B. P., \& Bushman, B. J. (2014). Development and validation of the single item narcissism scale (SINS). PLOS one, 9(8), e103469.

Tolmacz, R., \& Mikulincer, M. (2011). The sense of entitlement in romantic relationships-Scale construction, factor structure, construct validity, and its associations with attachment orientations. Psychoanalytic psychology, 28(1), 75-94. 